\title{
A POSTERIORI ERROR ESTIMATOR FOR LINEAR ELASTICITY BASED ON NONSYMMETRIC STRESS TENSOR APPROXIMATION
}

\author{
KWANG-YEON KIM
}

Department of Mathematics, Kangwon National University, Chuncheon, South Korea

E-mail address: eulerkimekangwon.ac.kr

\begin{abstract}
In this paper we present an a posteriori error estimator for the stabilized $P 1$ nonconforming finite element method of the linear elasticity problem based on a nonsymmetric $H$ (div)-conforming approximation of the stress tensor in the first-order Raviart-Thomas space. By combining the equilibrated residual method and the hypercircle method, it is shown that the error estimator gives a fully computable upper bound on the actual error. Numerical results are provided to confirm the theory and illustrate the effectiveness of our error estimator.
\end{abstract}

\section{INTRODUCTION}

In this paper we consider a homogeneous linear elastic material occupying a planar domain $\Omega$ whose displacement $\boldsymbol{u}: \Omega \rightarrow \mathbb{R}^{2}$ is described by the equations

$$
\sigma=\mathbb{C} \epsilon(u), \quad-\operatorname{div} \sigma=f \quad \text { in } \Omega
$$

subject to the boundary conditions

$$
\boldsymbol{u}=\boldsymbol{u}_{D} \quad \text { on } \Gamma_{D}, \quad \boldsymbol{\sigma n}=0 \quad \text { on } \Gamma_{N}:=\partial \Omega \backslash \Gamma_{D},
$$

where $\boldsymbol{\sigma}$ is the Cauchy stress tensor, $\boldsymbol{\epsilon}(\boldsymbol{u})=\frac{1}{2}\left(\nabla \boldsymbol{u}+(\nabla \boldsymbol{u})^{T}\right)$ is the linearized strain tensor, and $\boldsymbol{n}$ is the unit outward normal on $\partial \Omega$. For simplicity, we assume that $\Gamma_{D}$ is not empty, but it is easy to extend all subsequent results to the pure Neumann case.

The coefficient $\mathbb{C}$ is a fourth-order elasticity tensor which is bounded, uniformly positive definite and satisfies the symmetry condition. Hereafter we restrict ourselves to the isotropic material in a state of plane strain, in which case $\mathbb{C}$ is given in the form

$$
\mathbb{C} \boldsymbol{\epsilon}(\boldsymbol{u})=2 \mu \boldsymbol{\epsilon}(\boldsymbol{u})+\lambda \operatorname{div} \boldsymbol{u I},
$$

where $\boldsymbol{I}$ is the $2 \times 2$ identity tensor and $\mu, \lambda$ are the Lamé constants satisfying $0<\mu_{1}<\mu<\mu_{2}$ for some fixed $\mu_{1}, \mu_{2}$ and $0<\lambda<\infty$.

Received by the editors July 7 2011; Accepted December 232011.

2010 Mathematics Subject Classification. 65N30, 65N15.

Key words and phrases. a posteriori error estimator, linear elasticity, $P 1$ nonconforming finite element method.

This work was supported by the Korea Science and Engineering Foundation (KOSEF) grant funded by the Korea government (Grant No. 2009-0073414). 
The weak formulation for (1.1)-(1.2) seeks the displacement $\boldsymbol{u} \in \boldsymbol{H}_{D}^{1}\left(\Omega ; \boldsymbol{u}_{D}\right)$ such that

$$
(\mathbb{C} \boldsymbol{\epsilon}(\boldsymbol{u}), \boldsymbol{\epsilon}(\boldsymbol{v}))_{\Omega}=(\boldsymbol{f}, \boldsymbol{v})_{\Omega} \quad \forall \boldsymbol{v} \in \boldsymbol{H}_{D}^{1}(\Omega),
$$

where $(\cdot, \cdot)_{\Omega}$ is the standard inner product in $\left(L^{2}(\Omega)\right)^{d}(d=1,2)$ and

$$
\boldsymbol{H}_{D}^{1}\left(\Omega ; \boldsymbol{u}_{D}\right):=\left\{\boldsymbol{v} \in\left(H^{1}(\Omega)\right)^{2}:\left.\boldsymbol{v}\right|_{\Gamma_{D}}=\boldsymbol{u}_{D}\right\}, \quad \boldsymbol{H}_{D}^{1}(\Omega):=\boldsymbol{H}_{D}^{1}(\Omega ; 0) .
$$

We adopt the standard notation for the Sobolev space $H^{k}(S)$ over a set $S$ equipped with the norm $\|\cdot\|_{k, S}$ and semi-norm $|\cdot|_{k, S}$.

Nowadays it is well established that one should apply adaptive mesh refinement based on a posteriori error estimators for efficient implementation of numerical methods. Various types of error estimators have been developed and successfully implemented for the linear elasticity problem; see, for example, the survey paper [1]. We are particularly interested in the error estimators which give fully computable upper bounds on the actual error without involving unknown constants. Such error estimators were constructed in [2] for the $P 1$ conforming element and in [3] for the $P 2$ conforming and nonconforming elements by combining the equilibrated residual method and the hypercircle method. The key step there was recovery of a symmetric $H(\mathrm{div})$-conforming approximation of the stress tensor $\boldsymbol{\sigma}$ in an appropriate finite element space of symmetric tensors from the equilibrated normal stress approximation, which is quite complicated due to the large dimension of the local finite element space used (the Arnold-Winther space in [2] and the Arnold-Douglas-Gupta space in [3]), although the computation is done locally on each element.

In this paper we propose a new error estimator which requires much less computation than $[2,3]$, while achieving fully computable upper bounds on the actual error. This is accomplished by recovering a nonsymmetric $H(\mathrm{div})$-conforming approximation of the stress tensor $\boldsymbol{\sigma}$ in the nonsymmetric Raviart-Thomas space of first order whose local dimension is smaller and makes the implementation easier than the symmetric tensor spaces mentioned above. When compared with the ones from $[2,3]$, our estimator contains the additional contribution arising from the non-symmetry of the recovered stress tensor approximation and thus strongly depending on computable upper bounds on the constants of local Korn's inequality. A similar consideration was given to the Stokes problem in [4] but the derived estimator was not fully computable as the constant in the upper bound was not estimated.

To fix ideas, we consider the stabilized $P 1$ nonconforming finite element proposed in [5] for which, unlike the $P 1$ conforming element, the equilibrated normal stress approximation is explicitly constructed without solving local linear systems. It is straightforward to apply subsequent results to other $P 1$ and $P 2$ finite elements as long as the equilibrated normal stress approximation (defined in Section 3 ) is available.

The rest of the paper is organized as follows. In the next section we introduce the stabilized $P 1$ nonconforming finite element method and some computable estimates related to Korn's inequality. In Section 3 we construct the nonsymmetric $H$ (div)-conforming approximation of the stress tensor and then derive the a posteriori error estimator which yields an upper bound on the actual error in Section 4. Finally, Section 5 presents some numerical results to confirm the theory and illustrate the effectiveness of our error estimator. 


\section{StABILIZED $P 1$ NONCONFORMING FEM AND KoRN's INEQUALITY}

2.1. Stabilized $P 1$ Nonconforming FEM. Let $\mathcal{T}_{h}=\{T\}$ be a regular triangulation of $\Omega$ into triangles with $h_{T}=\operatorname{diam}(T)$ and $h=\max _{T \in \mathcal{T}_{h}} h_{T}$. The set of three edges of an element $T \in \mathcal{T}_{h}$ is denoted by $\mathcal{E}_{T}$ and the unit outward normal on $\partial T$ by $\boldsymbol{n}_{T}$.

Let $\mathcal{E}_{\Omega}, \mathcal{E}_{D}$ and $\mathcal{E}_{N}$ be the collections of all edges of $\mathcal{T}_{h}$ lying on $\Omega, \Gamma_{D}$ and $\Gamma_{N}$, respectively, and set $\mathcal{E}_{h}=\mathcal{E}_{\Omega} \cup \mathcal{E}_{D} \cup \mathcal{E}_{N}$. For each edge $E \in \mathcal{E}_{\Omega}$, we fix a unit normal vector $\boldsymbol{n}_{E}$ and define the jump of $\boldsymbol{v}$ across $E=\partial T^{+} \cap \partial T^{-}$as

$$
\left.\llbracket \boldsymbol{v} \rrbracket\right|_{E}=\left.\boldsymbol{v}\right|_{T^{+}}-\left.\boldsymbol{v}\right|_{T^{-}}
$$

where $\boldsymbol{n}_{E}$ is directed from $T^{+}$to $T^{-}$. For a boundary edge $E \in \mathcal{E}_{D} \cup \mathcal{E}_{N}, \boldsymbol{n}_{E}$ is taken to be outward to $\partial \Omega$ and $\left.\llbracket v \rrbracket\right|_{E}$ is set to be zero.

Let $\mathbb{P}_{k}(T)$ be the space of all polynomials on $T$ whose total degrees are less than or equal to $k$ and let $\mathcal{R} \mathcal{M}(T):=\operatorname{span}\{(1,0),(0,1),(y,-x)\}$ be the space of rigid body motions on $T$. Notice that $\boldsymbol{v} \in \mathcal{R} \mathcal{M}(T)$ if and only if $\boldsymbol{\epsilon}(\boldsymbol{v})=0$.

The Crouzeix-Raviart $P 1$ nonconforming finite element space is defined as

$$
\boldsymbol{V}_{h}=\left\{\boldsymbol{v}_{h} \in\left(L^{2}(\Omega)\right)^{2}:\left.\boldsymbol{v}_{h}\right|_{T} \in\left(\mathbb{P}_{1}(T)\right)^{2} \forall T \in \mathcal{T}_{h} \text { and } \int_{E} \llbracket \boldsymbol{v}_{h} \rrbracket d s=0 \quad \forall E \in \mathcal{E}_{\Omega}\right\},
$$

and the subspace of $\boldsymbol{V}_{h}$ with zero mean values on $\mathcal{E}_{D}$ as

$$
\boldsymbol{V}_{h, D}=\left\{\boldsymbol{v}_{h} \in \boldsymbol{V}_{h}: \int_{E} \boldsymbol{v}_{h} d s=0 \forall E \in \mathcal{E}_{D}\right\} .
$$

Then the stabilized $P 1$ nonconforming FEM for the linear elasticity problem (1.3) reads as follows (cf. [5]): find $\boldsymbol{u}_{h} \in \boldsymbol{V}_{h}$ such that $\int_{E}\left(\boldsymbol{u}_{h}-\boldsymbol{u}_{D}\right) d s=0$ for all $E \in \mathcal{E}_{D}$ and

$$
A_{h}\left(\boldsymbol{u}_{h}, \boldsymbol{v}_{h}\right)=\left(\boldsymbol{f}, \boldsymbol{v}_{h}\right)_{\Omega} \quad \forall \boldsymbol{v}_{h} \in \boldsymbol{V}_{h, D},
$$

where

$$
A_{h}\left(\boldsymbol{u}_{h}, \boldsymbol{v}_{h}\right)=\left(\mathbb{C} \boldsymbol{\epsilon}_{h}\left(\boldsymbol{u}_{h}\right), \boldsymbol{\epsilon}_{h}\left(\boldsymbol{v}_{h}\right)\right)_{\Omega}+\sum_{E \in \mathcal{E}_{\Omega}} \mu \gamma h_{E}^{-1} \int_{E} \llbracket \boldsymbol{u}_{h} \rrbracket \cdot \llbracket \boldsymbol{v}_{h} \rrbracket d s .
$$

Here $\gamma>0$ is the stabilization parameter, $h_{E}=\operatorname{diam}(E)$, and $\epsilon_{h}(\cdot)$ is the differential operator $\boldsymbol{\epsilon}(\cdot)$ applied piecewise over $\mathcal{T}_{h}$.

2.2. Korn's Inequality and related estimates. For a subdomain $K \subset \Omega$, Korn's inequality asserts that there exists a constant $R_{K}>0$ depending only on $K$ such that

$$
\|\nabla \boldsymbol{v}\|_{0, K}^{2} \leq R_{K}\|\boldsymbol{\epsilon}(\boldsymbol{v})\|_{0, K}^{2}
$$

for all $\boldsymbol{v} \in\left(H^{1}(K)\right)^{2}$ with $\int_{K} \operatorname{curl} \boldsymbol{v} d \boldsymbol{x}=0$. Using the results in [6, 7], one can obtain the following computable upper bounds

$$
R_{T} \leq \frac{2}{\sin ^{2}\left(\theta_{\min } / 4\right)}, \quad R_{S} \leq \frac{2}{\sin ^{2}(\pi / 8)}=13.6569 \ldots
$$

for a triangle $T$ with the minimum angle $\theta_{\min }$ and a square $S$ (see [8]). 
In deriving the a posteriori error estimator based on a nonsymmetric approximation of the stress tensor, we will need the operator $\Pi_{K}:\left(H^{1}(K)\right)^{2} \rightarrow \mathcal{R} \mathcal{M}(K)$ defined by

$$
\int_{K}\left(\boldsymbol{v}-\Pi_{K} \boldsymbol{v}\right) d x=\int_{K} \operatorname{curl}\left(\boldsymbol{v}-\Pi_{K} \boldsymbol{v}\right) d x=0 .
$$

The following estimates were first noted in [3, Appendix A]

$$
\begin{aligned}
& \left\|\nabla\left(\boldsymbol{v}-\Pi_{K} \boldsymbol{v}\right)\right\|_{0, K} \leq R_{K}^{1 / 2}\|\boldsymbol{\epsilon}(\boldsymbol{v})\|_{0, K}, \\
& \left\|\boldsymbol{v}-\Pi_{K} \boldsymbol{v}\right\|_{0, K} \leq R_{K}^{1 / 2} \frac{h_{K}}{\pi}\|\boldsymbol{\epsilon}(\boldsymbol{v})\|_{0, K},
\end{aligned}
$$

where $h_{K}$ is the diameter of $K$. The first result is a direct consequence of Korn's inequality (2.2) and the second result can be obtained by applying the Poincaré inequality [9] and then the first result.

\section{3. $H$ (div)-CONFORMing Approximation OF STRESS TENSOR}

To recover a $H$ (div)-conforming approximation of the stress tensor $\boldsymbol{\sigma}$, we first need to construct the normal stress approximation $\left.\boldsymbol{g}_{T} \approx \boldsymbol{\sigma}_{T}\right|_{\partial T}$ on every element $T \in \mathcal{T}_{h}$ which fulfills the following equilibration conditions (see, e.g., $[2,10,11]$ )

$$
\begin{array}{rlrl}
\left.\boldsymbol{g}_{T_{1}}\right|_{E}+\left.\boldsymbol{g}_{T_{2}}\right|_{E} & =0 & & \text { for } E=\partial T_{1} \cap \partial T_{2}, \\
\left.\boldsymbol{g}_{T}\right|_{E} & =0 & & \text { for } E \in \mathcal{E}_{T} \cap \mathcal{E}_{N}, \\
\int_{T} \boldsymbol{f} \cdot \boldsymbol{v} d \boldsymbol{x}+\int_{\partial T} \boldsymbol{g}_{T} \cdot \boldsymbol{v} d s=0 & \text { for } \boldsymbol{v} \in \mathcal{R} \mathcal{M}(T) .
\end{array}
$$

For the stabilized $P 1$ nonconforming FEM (2.1), this can be done by an easy adaptation of the result for the Stokes problem given in [4].

Definition 3.1. For each $T \in \mathcal{T}_{h}$ and $E \in \mathcal{E}_{T}$, we define the normal stress approximation

$$
\begin{aligned}
\left.\boldsymbol{g}_{T}\right|_{E}=\frac{1}{|E|}\left\{\int_{T} \mathbb{C} \boldsymbol{\epsilon}\left(\boldsymbol{u}_{h}\right) \nabla \phi_{E}^{(T)}\right. & d \boldsymbol{x}-\int_{T} \boldsymbol{f} \phi_{E}^{(T)} d \boldsymbol{x} \\
& \left.+\sum_{E^{\prime} \in \mathcal{E}_{T}} \mu \gamma h_{E^{\prime}}^{-1} \int_{E^{\prime}} \llbracket \boldsymbol{u}_{h} \rrbracket_{\partial T} \phi_{E}^{(T)} d s\right\}-\left.\mu \gamma h_{E}^{-1} \llbracket \boldsymbol{u}_{h} \rrbracket_{\partial T}\right|_{E},
\end{aligned}
$$

where $\llbracket \boldsymbol{v} \rrbracket_{\partial T}$ is the jump of $\boldsymbol{v}$ from the interior to the exterior of $T$

$$
\left.\llbracket \boldsymbol{v} \rrbracket_{\partial T}\right|_{E}=\left.\left(\left.\boldsymbol{n}_{T}\right|_{E} \cdot \boldsymbol{n}_{E}\right) \llbracket \boldsymbol{v} \rrbracket\right|_{E} .
$$

Here $\phi_{E}^{(T)}$ denotes the scalar-valued local basis function for $\mathbb{P}_{1}(T)$ associated with the edge $E \in \mathcal{E}_{T}$ satisfying

$$
\int_{E^{\prime}} \phi_{E}^{(T)} d s=\delta_{E, E^{\prime}}|E| \quad \forall E^{\prime} \in \mathcal{E}_{T}
$$

The proof of the equilibration conditions (3.1)-(3.3) can be done similarly to [4, Theorem 1]. 
Now we recover a nonsymmetric $H$ (div)-conforming approximation $\boldsymbol{\sigma}_{h}$ of the stress tensor in the nonsymmetric Raviart-Thomas space of first order from the equilibrated normal stress approximation $\boldsymbol{g}_{T}$ given in Definition 3.1 which is piecewise linear over $\partial T$. We remark that the same recovery process was exploited in [12] for a posteriori error estimation of the Stokes equation involving the nonsymmetric gradient tensor. Below $P_{k}$ denotes the $L^{2}$ projection onto the space of piecewise constant $(k=0)$ or linear $(k=1)$ functions over $\mathcal{T}_{h}$.

Definition 3.2. For each element $T \in \mathcal{T}_{h}$, we determine $\left.\boldsymbol{\sigma}_{h}^{1}\right|_{T} \in\left(\mathbb{P}_{1}(T)\right)^{2 \times 2}$ by the condition

$$
\left.\boldsymbol{\sigma}_{h}^{1}\right|_{T} \boldsymbol{n}_{T}=\boldsymbol{g}_{T} \text { on } \partial T
$$

and define the stress tensor approximation

$$
\left.\boldsymbol{\sigma}_{h}\right|_{T}=\left.\boldsymbol{\sigma}_{h}^{1}\right|_{T}-\frac{1}{3} \sum_{i=1}^{3} P_{1} \boldsymbol{f}\left(\boldsymbol{x}_{i}\right) \otimes\left(\boldsymbol{x}-\boldsymbol{x}_{i}\right) \lambda_{i},
$$

where $\left\{\boldsymbol{x}_{i}\right\}_{i=1,2,3}$ are the vertices of $T,\left\{\lambda_{i}\right\}_{i=1,2,3}$ are the barycentric coordinates such that $\lambda_{i}\left(\boldsymbol{x}_{j}\right)=\delta_{i j}$, and $(\boldsymbol{u} \otimes \boldsymbol{v})_{i j}:=u_{i} v_{j}$.

Since $\left(\boldsymbol{x}-\boldsymbol{x}_{i}\right) \lambda_{i}$ has vanishing normal components on $\partial T$, we immediately get

$$
\left.\boldsymbol{\sigma}_{h}\right|_{T} \boldsymbol{n}_{T}=\boldsymbol{g}_{T} \quad \text { on } \partial T,
$$

from which it follows by (3.1)-(3.2) that $\boldsymbol{\sigma}_{h}$ is indeed $H(\operatorname{div})$-conforming and $\left.\boldsymbol{\sigma}_{h} \cdot \boldsymbol{n}\right|_{\Gamma_{N}}=0$. Moreover, we have the following result.

Theorem 3.3. Let $\boldsymbol{\sigma}_{h}$ be defined by Definition 3.2. Then we have

$$
\operatorname{div} \boldsymbol{\sigma}_{h}+P_{1} \boldsymbol{f}=0 \quad \text { and } \quad P_{0} \boldsymbol{\sigma}_{h}^{A S}=0 .
$$

Proof. The proof of the first result is essentially given in [12]. We recall the proof for the reader's convenience. Taking $\boldsymbol{v} \in\left(\mathbb{P}_{0}(T)\right)^{2}$ in (3.3), we can show that

$$
\operatorname{div} \boldsymbol{\sigma}_{h}^{1}+P_{0} \boldsymbol{f}=0 .
$$

Now use the identity

$$
\lambda_{i}(\boldsymbol{x})=\nabla \lambda_{i} \cdot\left(\boldsymbol{x}-\boldsymbol{x}_{i}\right)+1
$$

to obtain

$$
\begin{aligned}
\operatorname{div} \boldsymbol{\sigma}_{h} & =\operatorname{div} \boldsymbol{\sigma}_{h}^{1}-\frac{1}{3} \sum_{i=1}^{3} P_{1} \boldsymbol{f}\left(\boldsymbol{x}_{i}\right) \operatorname{div}\left(\left(\boldsymbol{x}-\boldsymbol{x}_{i}\right) \lambda_{i}\right) \\
& =-P_{0} \boldsymbol{f}-\frac{1}{3} \sum_{i=1}^{3} P_{1} \boldsymbol{f}\left(\boldsymbol{x}_{i}\right)\left(3 \lambda_{i}-1\right) \\
& =-P_{0} \boldsymbol{f}-\left(P_{1} \boldsymbol{f}-P_{0} \boldsymbol{f}\right)=-P_{1} \boldsymbol{f}
\end{aligned}
$$


which is the first result. To prove the second result, we use (3.4), the first result and then (3.3) to obtain for $\boldsymbol{v}_{h} \in \mathcal{R} \mathcal{M}(T)$

$$
\begin{aligned}
\int_{T} \boldsymbol{\sigma}_{h}: \nabla \boldsymbol{v}_{h} d \boldsymbol{x} & =\int_{\partial T} \boldsymbol{\sigma}_{h} \boldsymbol{n}_{T} \cdot \boldsymbol{v}_{h} d s-\int_{T} \operatorname{div} \boldsymbol{\sigma}_{h} \cdot \boldsymbol{v}_{h} d \boldsymbol{x} \\
& =\int_{\partial T} \boldsymbol{g}_{T} \cdot \boldsymbol{v}_{h} d s+\int_{T} \boldsymbol{f} \cdot \boldsymbol{v}_{h} d \boldsymbol{x}=0 .
\end{aligned}
$$

Now the second result is obtained by taking $\boldsymbol{v}_{h}=(y,-x)$.

\section{A POSTERIORI ERror Estimation}

In this section we will derive and analyze an a posteriori error estimator which gives a fully computable upper bound on the numerical error $\boldsymbol{u}-\boldsymbol{u}_{h}$ of the $P 1$ nonconforming FEM (2.1) measured in the energy semi-norm

$$
\|\boldsymbol{v}\|^{2}:=\left(\mathbb{C} \boldsymbol{\epsilon}_{h}(\boldsymbol{v}), \boldsymbol{\epsilon}_{h}(\boldsymbol{v})\right)_{\Omega}=\sum_{T \in \mathcal{T}_{h}}\|\boldsymbol{v}\|_{T}^{2}, \quad\|\boldsymbol{v}\|_{T}^{2}:=(\mathbb{C} \boldsymbol{\epsilon}(\boldsymbol{v}), \boldsymbol{\epsilon}(\boldsymbol{v}))_{T} .
$$

Following [3, 4], we decompose the error $\boldsymbol{u}-\boldsymbol{u}_{h}$ into two contributions

$$
\boldsymbol{u}-\boldsymbol{u}_{h}=(\boldsymbol{u}-\boldsymbol{\xi})+\left(\boldsymbol{\xi}-\boldsymbol{u}_{h}\right)
$$

where $\boldsymbol{\xi} \in \boldsymbol{H}_{D}^{1}\left(\Omega ; \boldsymbol{u}_{D}\right)$ is the solution of

$$
(\mathbb{C} \boldsymbol{\epsilon}(\boldsymbol{\xi}), \boldsymbol{\epsilon}(\boldsymbol{v}))_{\Omega}=\left(\mathbb{C} \boldsymbol{\epsilon}_{h}\left(\boldsymbol{u}_{h}\right), \boldsymbol{\epsilon}(\boldsymbol{v})\right)_{\Omega} \quad \forall \boldsymbol{v} \in \boldsymbol{H}_{D}^{1}(\Omega) .
$$

By the Galerkin orthogonality $\left(\mathbb{C} \epsilon_{h}\left(\boldsymbol{\xi}-\boldsymbol{u}_{h}\right), \boldsymbol{\epsilon}(\boldsymbol{u}-\boldsymbol{\xi})\right)_{\Omega}=0$, we obtain the Pythagorean relationship

$$
\left\|\boldsymbol{u}-\boldsymbol{u}_{h}\right\|^{2}=\|\boldsymbol{u}-\boldsymbol{\xi}\|^{2}+\left\|\boldsymbol{\xi}-\boldsymbol{u}_{h}\right\|^{2} .
$$

The following lemma presents the abstract error formulas for the two contributions on the right-hand side, the proof of which is a simple modification of that of [4].

Lemma 4.1. Let $\boldsymbol{\xi} \in \boldsymbol{H}_{D}^{1}\left(\Omega ; \boldsymbol{u}_{D}\right)$ be the solution of (4.1). Then we have

$$
\|\boldsymbol{u}-\boldsymbol{\xi}\|=\sup _{\boldsymbol{v} \in \boldsymbol{H}_{D}^{1}(\Omega)} \frac{(\boldsymbol{f}, \boldsymbol{v})_{\Omega}-\left(\mathbb{C} \boldsymbol{\epsilon}_{h}\left(\boldsymbol{u}_{h}\right), \boldsymbol{\epsilon}(\boldsymbol{v})\right)_{\Omega}}{\|\boldsymbol{v}\|}
$$

and

$$
\left\|\boldsymbol{\xi}-\boldsymbol{u}_{h}\right\|=\inf _{\boldsymbol{\chi} \in \boldsymbol{H}_{D}^{1}\left(\Omega ; \boldsymbol{u}_{D}\right)}\left\|\boldsymbol{\chi}-\boldsymbol{u}_{h}\right\| .
$$

Proof. The first result is an immediate consequence of the equality

$$
(\mathbb{C} \boldsymbol{\epsilon}(\boldsymbol{u}-\boldsymbol{\xi}), \boldsymbol{\epsilon}(\boldsymbol{v}))_{\Omega}=(\boldsymbol{f}, \boldsymbol{v})_{\Omega}-\left(\mathbb{C} \boldsymbol{\epsilon}_{h}\left(\boldsymbol{u}_{h}\right), \boldsymbol{\epsilon}(\boldsymbol{v})\right)_{\Omega} \quad \forall \boldsymbol{v} \in \boldsymbol{H}_{D}^{1}(\Omega),
$$

while the second result follows from the fact that $\boldsymbol{\xi}$ is the orthogonal projection of $\boldsymbol{u}_{h}$ onto the hyperplane $\boldsymbol{H}_{D}^{1}\left(\Omega ; \boldsymbol{u}_{D}\right)$ with respect to the energy semi-inner product $\left(\mathbb{C} \boldsymbol{\epsilon}_{h}(\cdot), \boldsymbol{\epsilon}_{h}(\cdot)\right)_{\Omega}$.

By the estimate (4.3), $\left\|\boldsymbol{\xi}-\boldsymbol{u}_{h}\right\|$ measures the distance between the numerical solution $\boldsymbol{u}_{h}$ and the continuous solution space $\boldsymbol{H}_{D}^{1}\left(\Omega ; \boldsymbol{u}_{D}\right)$ in the energy semi-norm, commonly referred to as the nonconforming error, while $\|\boldsymbol{u}-\boldsymbol{\xi}\|$ is called the conforming error. 
4.1. Conforming error estimator. In this subsection we combine the equilibrated residual method and the hypercircle method to derive an estimator for the conforming error $\|\boldsymbol{u}-\boldsymbol{\xi}\|$ based on the estimate (4.2). To begin with, let us define the symmetric and antisymmetric parts of a tensor $\boldsymbol{\tau}=\boldsymbol{\tau}^{S}+\boldsymbol{\tau}^{A S}$ by

$$
\boldsymbol{\tau}^{S}=\frac{\boldsymbol{\tau}+\boldsymbol{\tau}^{T}}{2} \quad \text { and } \quad \boldsymbol{\tau}^{A S}=\frac{\boldsymbol{\tau}-\boldsymbol{\tau}^{T}}{2},
$$

where $\tau^{T}$ is the transpose of $\tau$. It is obvious that

$$
\left(\boldsymbol{\tau}^{S}, \boldsymbol{\tau}^{A S}\right)_{T}=0 \quad \text { and } \quad\left\|\boldsymbol{\tau}^{S}\right\|_{0, T}+\left\|\boldsymbol{\tau}^{A S}\right\|_{0, T} \leq\|\boldsymbol{\tau}\|_{0, T} .
$$

Now we present the main result of this paper which generalizes Theorem 3.4 in [2] to the nonsymmetric $H$ (div)-conforming approximation of the stress tensor.

Theorem 4.2. Let $\sigma_{h}$ be defined by Definition 3.2 and let

$$
\begin{aligned}
\eta_{C F, T} & :=\left\|\mathbb{C}^{-1 / 2}\left(\boldsymbol{\sigma}_{h}^{S}-\mathbb{C} \boldsymbol{\epsilon}\left(\boldsymbol{u}_{h}\right)\right)\right\|_{0, T}+\left(\frac{R_{T}}{2 \mu}\right)^{1 / 2} \frac{h_{T}}{\pi}\left\|\boldsymbol{f}+\operatorname{div} \boldsymbol{\sigma}_{h}\right\|_{0, T} \\
\eta_{A S, K} & :=\left(\frac{R_{K}}{2 \mu}\right)^{1 / 2}\left\|\boldsymbol{\sigma}_{h}^{A S}\right\|_{0, K}
\end{aligned}
$$

for an element $T$ and a subdomain $K$. Then we have

$$
\|\boldsymbol{u}-\boldsymbol{\xi}\| \leq\left\{\sum_{T \in \mathcal{T}_{h}}\left(\eta_{C F, T}+\eta_{A S, T}\right)^{2}\right\}^{1 / 2} .
$$

Moreover, if $\Omega$ is partitioned into the subdomains $\left\{\Omega_{i}\right\}_{i=1}^{N}$ such that each $\Omega_{i}$ is a union of elements in $\mathcal{T}_{h}$, then we have

$$
\|\boldsymbol{u}-\boldsymbol{\xi}\| \leq\left(\sum_{T \in \mathcal{T}_{h}} \eta_{C F, T}^{2}\right)^{1 / 2}+\left(\sum_{i=1}^{N} \eta_{A S, \Omega_{i}}^{2}\right)^{1 / 2}
$$

Proof. We are going to estimate the right-hand side of (4.2). First note that for $\boldsymbol{v} \in \boldsymbol{H}_{D}^{1}(\Omega)$,

$$
(\boldsymbol{f}, \boldsymbol{v})_{\Omega}-\left(\mathbb{C} \boldsymbol{\epsilon}_{h}\left(\boldsymbol{u}_{h}\right), \boldsymbol{\epsilon}(\boldsymbol{v})\right)_{\Omega}=\sum_{T \in \mathcal{T}_{h}} r_{T}(\boldsymbol{v}),
$$

where

$$
r_{T}(\boldsymbol{v}):=\int_{T} \boldsymbol{f} \cdot \boldsymbol{v} d \boldsymbol{x}+\int_{\partial T} \boldsymbol{\sigma}_{h} \boldsymbol{n}_{T} \cdot \boldsymbol{v} d s-\int_{T} \mathbb{C} \boldsymbol{\epsilon}\left(\boldsymbol{u}_{h}\right): \boldsymbol{\epsilon}(\boldsymbol{v}) d \boldsymbol{x} .
$$

Using integration by parts, we get

$$
\begin{aligned}
r_{T}(\boldsymbol{v}) & =\int_{T} \boldsymbol{\sigma}_{h}: \nabla \boldsymbol{v} d \boldsymbol{x}-\int_{T} \mathbb{C} \boldsymbol{\epsilon}\left(\boldsymbol{u}_{h}\right): \boldsymbol{\epsilon}(\boldsymbol{v}) d \boldsymbol{x}+\int_{T}\left(\boldsymbol{f}+\operatorname{div} \boldsymbol{\sigma}_{h}\right) \cdot \boldsymbol{v} d \boldsymbol{x} \\
& =\int_{T}\left(\boldsymbol{\sigma}_{h}^{S}-\mathbb{C} \boldsymbol{\epsilon}\left(\boldsymbol{u}_{h}\right)\right): \boldsymbol{\epsilon}(\boldsymbol{v}) d \boldsymbol{x}+\int_{T}\left(\boldsymbol{f}+\operatorname{div} \boldsymbol{\sigma}_{h}\right) \cdot \boldsymbol{v} d \boldsymbol{x}+\int_{T} \boldsymbol{\sigma}_{h}^{A S}: \nabla \boldsymbol{v} d \boldsymbol{x} .
\end{aligned}
$$


The first term is simply bounded by

$$
\int_{T}\left(\boldsymbol{\sigma}_{h}^{S}-\mathbb{C} \boldsymbol{\epsilon}\left(\boldsymbol{u}_{h}\right)\right): \boldsymbol{\epsilon}(\boldsymbol{v}) d \boldsymbol{x} \leq\left\|\mathbb{C}^{-1 / 2}\left(\boldsymbol{\sigma}_{h}^{S}-\mathbb{C} \boldsymbol{\epsilon}\left(\boldsymbol{u}_{h}\right)\right)\right\|_{0, T}\|\boldsymbol{v}\|_{T} .
$$

The second term is handled by using the first result of (3.5) and the estimate (2.5)

$$
\begin{aligned}
\int_{T}\left(\boldsymbol{f}+\operatorname{div} \boldsymbol{\sigma}_{h}\right) \cdot \boldsymbol{v} d \boldsymbol{x} & =\int_{T}\left(\boldsymbol{f}+\operatorname{div} \boldsymbol{\sigma}_{h}\right) \cdot\left(\boldsymbol{v}-\Pi_{T} \boldsymbol{v}\right) d \boldsymbol{x} \\
& \leq\left\|\boldsymbol{f}+\operatorname{div} \boldsymbol{\sigma}_{h}\right\|_{0, T} \cdot R_{T}^{1 / 2} \frac{h_{T}}{\pi}\|\boldsymbol{\epsilon}(\boldsymbol{v})\|_{0, T} \\
& \leq\left(\frac{R_{T}}{2 \mu}\right)^{1 / 2} \frac{h_{T}}{\pi}\left\|\boldsymbol{f}+\operatorname{div} \boldsymbol{\sigma}_{h}\right\|_{0, T}\|\boldsymbol{v}\|_{T},
\end{aligned}
$$

where we used the inequality $\|\boldsymbol{\epsilon}(\boldsymbol{v})\|_{0, T}^{2} \leq \frac{1}{2 \mu}\|\boldsymbol{v}\|_{T}^{2}$. As a result, it follows that

$$
\int_{T}\left(\boldsymbol{\sigma}_{h}^{S}-\mathbb{C} \boldsymbol{\epsilon}\left(\boldsymbol{u}_{h}\right)\right): \boldsymbol{\epsilon}(\boldsymbol{v}) d \boldsymbol{x}+\int_{T}\left(\boldsymbol{f}+\operatorname{div} \boldsymbol{\sigma}_{h}\right) \cdot \boldsymbol{v} d \boldsymbol{x} \leq \eta_{C F, T}\|\boldsymbol{v}\|_{T} .
$$

For the third term, we use the second result of (3.5) and the estimate (2.4) on each element $T$ to obtain

$$
\int_{T} \boldsymbol{\sigma}_{h}^{A S}: \nabla \boldsymbol{v} d \boldsymbol{x}=\int_{T} \boldsymbol{\sigma}_{h}^{A S}: \nabla\left(\boldsymbol{v}-\Pi_{T} \boldsymbol{v}\right) d \boldsymbol{x} \leq\left(\frac{R_{T}}{2 \mu}\right)^{1 / 2}\left\|\boldsymbol{\sigma}_{h}^{A S}\right\|_{0, T}\|\boldsymbol{v}\|_{T},
$$

which gives

$$
\sum_{T \in \mathcal{T}_{h}} r_{T}(\boldsymbol{v}) \leq \sum_{T \in \mathcal{T}_{h}}\left(\eta_{C F, T}+\eta_{A S, T}\right)\|\boldsymbol{v}\|_{T} \leq\left\{\sum_{T \in \mathcal{T}_{h}}\left(\eta_{C F, T}+\eta_{A S, T}\right)^{2}\right\}^{1 / 2}\|\boldsymbol{v}\| .
$$

Otherwise we can do the same thing on each subdomain $\Omega_{i}$ to obtain

$$
\int_{\Omega_{i}} \boldsymbol{\sigma}_{h}^{A S}: \nabla \boldsymbol{v} d \boldsymbol{x}=\int_{\Omega_{i}} \boldsymbol{\sigma}_{h}^{A S}: \nabla\left(\boldsymbol{v}-\Pi_{\Omega_{i}} \boldsymbol{v}\right) d \boldsymbol{x} \leq\left(\frac{R_{\Omega_{i}}}{2 \mu}\right)^{1 / 2}\left\|\boldsymbol{\sigma}_{h}^{A S}\right\|_{0, \Omega_{i}}\|\boldsymbol{v}\|_{\Omega_{i}},
$$

which gives

$$
\begin{aligned}
\sum_{T \in \mathcal{T}_{h}} r_{T}(\boldsymbol{v}) & \leq \sum_{T \in \mathcal{T}_{h}} \eta_{C F, T}\|\boldsymbol{v}\|_{T}+\sum_{i=1}^{N} \eta_{A S, \Omega_{i}}\|\boldsymbol{v}\|_{\Omega_{i}} \\
& \leq\left(\sum_{T \in \mathcal{T}_{h}} \eta_{C F, T}^{2}\right)^{1 / 2}\|\boldsymbol{v}\|+\left(\sum_{i=1}^{N} \eta_{A S, \Omega_{i}}^{2}\right)^{1 / 2}\|\boldsymbol{v}\| .
\end{aligned}
$$

The proof is completed by invoking the estimate (4.2).

Remark 4.3. One can readily check that Theorem 4.2 is valid for any $H$ (div)-conforming approximation $\sigma_{h}$ satisfying (3.5). In particular, the symmetric approximation of the stress tensor from $\boldsymbol{g}_{T}$ in the Arnold-Winther space [2] or in the Arnold-Douglas-Gupta space [3] yields a simpler error estimator with $\sigma_{h}^{A S}=0$. But the nonsymmetric approximation given in 
Definition 3.2 requires much less computation and makes the implementation easier than the symmetric ones.

Remark 4.4. In practice, $\eta_{C F, T}$ and $\eta_{A S, K}$ are computed using the upper bounds (2.3). The second result of Theorem 4.2 may give sharper upper bounds, e.g., when every $\Omega_{i}$ is a square.

4.2. Nonconforming error estimator. Based on the estimate (4.3), a computable upper bound on the nonconforming error $\left\|\boldsymbol{\xi}-\boldsymbol{u}_{h}\right\|$ is obtained by choosing a suitable $\boldsymbol{\chi} \in \boldsymbol{H}_{D}^{1}\left(\Omega ; \boldsymbol{u}_{D}\right)$. Like in [3,4], we simply choose the continuous piecewise linear or quadratic polynomial $\widetilde{\boldsymbol{u}}_{h}$ constructed by averaging the nodal values of $\boldsymbol{u}_{h}$ at Lagrange nodes and enforcing the Dirichlet boundary condition at Dirichlet nodes, i.e., interpolating

$$
\widetilde{\boldsymbol{u}}_{h}(\boldsymbol{z})=\left\{\begin{array}{cl}
\left.\frac{1}{\operatorname{card}\left(\omega_{\boldsymbol{z}}\right)} \sum_{T \in \omega_{\boldsymbol{z}}} \boldsymbol{u}_{h}\right|_{T}(\boldsymbol{z}) & \text { for } \boldsymbol{z} \in \mathcal{N}_{h} \backslash \mathcal{N}_{h, D}, \\
\boldsymbol{u}_{D}(\boldsymbol{z}) & \text { for } \boldsymbol{z} \in \mathcal{N}_{h, D}
\end{array}\right.
$$

where $\mathcal{N}_{h}$ is the set of all linear or quadratic Lagrange nodes of $\mathcal{T}_{h}, \mathcal{N}_{h, D}=\mathcal{N}_{h} \cap \Gamma_{D}$, and $\omega_{\boldsymbol{z}}$ is the set of all elements in $\mathcal{T}_{h}$ sharing the node $z$. The proof of the following theorem is trivial and thus omitted.

Theorem 4.5. Let $\widetilde{\boldsymbol{u}}_{h}$ be the continuous piecewise linear or quadratic polynomial constructed as above and let

$$
\eta_{N C, T}:=\left\|\widetilde{\boldsymbol{u}}_{h}-\boldsymbol{u}_{h}\right\|_{T}, \quad \operatorname{osc}_{T}\left(\boldsymbol{u}_{D}\right):=\inf _{\chi}\|\chi\|,
$$

where $\widetilde{\boldsymbol{u}}_{D}:=\left.\widetilde{\boldsymbol{u}}_{h}\right|_{\Gamma_{D}}$ and the infimum is taken over all $\chi \in \boldsymbol{H}^{1}(T)$ such that

$$
\chi=\left\{\begin{array}{cl}
\boldsymbol{u}_{D}-\widetilde{\boldsymbol{u}}_{D} & \text { on } \partial T \cap \Gamma_{D}, \\
0 & \text { on } \partial T \backslash \Gamma_{D} .
\end{array}\right.
$$

Then we have

$$
\left\|\boldsymbol{\xi}-\boldsymbol{u}_{h}\right\| \leq\left\{\sum_{T \in \mathcal{T}_{h}}\left(\eta_{N C, T}+\operatorname{osc}_{T}\left(\boldsymbol{u}_{D}\right)\right)^{2}\right\}^{1 / 2} .
$$

Remark 4.6. Since $\widetilde{\boldsymbol{u}}_{D}$ is the piecewise linear or quadratic Lagrange interpolant of $\boldsymbol{u}_{D}$ over $\mathcal{E}_{D}$, the second term $\operatorname{osc}_{T}\left(\boldsymbol{u}_{D}\right)$ is called the data oscillation of $\boldsymbol{u}_{D}$ on $\partial T \cap \Gamma_{D}$ and it can be shown that $(c f .[3,4])$

$$
\operatorname{osc}_{T}\left(\boldsymbol{u}_{D}\right) \leq C\left(\sum_{E \in \mathcal{E}_{T} \cap \mathcal{E}_{D}} h_{E}\left|\boldsymbol{u}_{D}-\widetilde{\boldsymbol{u}}_{D}\right|_{1, E}^{2}\right)^{1 / 2} .
$$

This term can be estimated using an extension of the boundary data (4.4) as in [3] or may be regarded as a higher order perturbation and negligible if $\boldsymbol{u}_{D}$ is piecewise smooth over $\mathcal{E}_{D}$. 


\section{NUMERICAL RESUlts}

In this section we present some numerical results to confirm the theory established in the previous section and illustrate the effectiveness of our error estimator. Since the domain $\Omega$ is either a square (Example 1) or composed of three squares which are unions of elements in $\mathcal{T}_{h}$ (Example 2), we can define the total error estimator in two ways (cf. Theorem 4.2)

$$
\eta_{0}^{2}:=\sum_{T \in \mathcal{T}_{h}}\left(\eta_{C F, T}+\eta_{A S, T}\right)^{2}+\sum_{T \in \mathcal{T}_{h}} \eta_{N C, T}^{2}
$$

and

$$
\eta_{1}^{2}:=\left\{\left(\sum_{T \in \mathcal{T}_{h}} \eta_{C F, T}^{2}\right)^{1 / 2}+\left(\frac{R_{S}}{2 \mu}\right)^{1 / 2}\left\|\boldsymbol{\sigma}_{h}^{A S}\right\|_{0, \Omega}\right\}^{2}+\sum_{T \in \mathcal{T}_{h}} \eta_{N C, T}^{2},
$$

where the constants $R_{T}$ and $R_{S}$ are replaced by the upper bounds given in (2.3), respectively, and the data oscillation $\operatorname{osc}_{T}\left(\boldsymbol{u}_{D}\right)$ is ignored. The nonconforming error estimator $\eta_{N C, T}$ is computed using the piecewise quadratic polynomial. For comparison we also compute the total error estimator

$$
\eta_{\text {sym }}^{2}:=\sum_{T \in \mathcal{T}_{h}} \eta_{C F, T}^{2}+\sum_{T \in \mathcal{T}_{h}} \eta_{N C, T}^{2}
$$

using the symmetric approximation of the stress tensor in the Arnold-Winther space (cf. [2]).

Example 1. Consider the following solution $\boldsymbol{u}=\left(u_{1}, u_{2}\right)$ on the unit square $\Omega=(0,1)^{2}$

$$
u_{1}(x, y)=\cos (2 \pi x) \sin (2 \pi y), \quad u_{2}(x, y)=-u_{1}(y, x)
$$

with the Lamé constants $\mu=1.0, \lambda=5.0$ and the corresponding body force $f$. We impose the homogeneous Neumann boundary condition

$$
\boldsymbol{\sigma n}=0 \quad \text { on } \Gamma_{N}=\{(x, y): x=1,0 \leq y \leq 1\} .
$$

and the Dirichlet boundary condition on the remaining part $\Gamma_{D}=\partial \Omega \backslash \Gamma_{N}$.

As the solution $\boldsymbol{u}$ is smooth, numerical experiments are performed on a sequence of uniform meshes obtained by first partitioning $\Omega$ into equal squares of size $h=\frac{1}{2^{m}}(m=3,4,5,6,7,8)$ and then dividing every square into two triangles along the diagonal of slope 1.

Numerical results are reported in Tables 1-2, where the three contributions of the total error estimator are defined as

$$
\eta_{C F}^{2}=\sum_{T \in \mathcal{T}_{h}} \eta_{C F, T}^{2}, \quad \eta_{A S}^{2}=\sum_{T \in \mathcal{T}_{h}} \eta_{A S, T}^{2} \quad \text { or } \frac{R_{S}}{2 \mu}\left\|\boldsymbol{\sigma}_{h}^{A S}\right\|_{0, \Omega}^{2}, \quad \eta_{N C}^{2}=\sum_{T \in \mathcal{T}_{h}} \eta_{N C, T}^{2}
$$

and the effectivity index $\theta$ is the ratio of the total error estimator to the actual error $\left\|\boldsymbol{u}-\boldsymbol{u}_{h}\right\|$. We observe that $\theta$ is always bigger than unity, which means that the total error estimator gives an upper bound on the actual error as predicted by the theory. Although the best result is obtained for the estimator $\eta_{s y m}$, our estimator $\eta_{1}$ seems to be competitive in terms of accuracy and computational cost. This is partly due to the fact that the common contribution $\eta_{N C}$ is comparable to the sum $\eta_{C F}+\eta_{A S}$. 
TABLE 1. Mesh size, actual error, three contributions of $\eta_{1}$ and effectivity index for Example 1.

\begin{tabular}{rccccc}
\hline $1 / h$ & $\left\|\boldsymbol{u}-\boldsymbol{u}_{h}\right\|$ & $\eta_{C F}$ & $\eta_{A S}$ & $\eta_{N C}$ & $\theta$ \\
\hline 8 & $1.802 \mathrm{e}+0$ & $1.573 \mathrm{e}+0$ & $1.997 \mathrm{e}+0$ & $3.025 \mathrm{e}+0$ & 2.597 \\
16 & $8.998 \mathrm{e}-1$ & $5.631 \mathrm{e}-1$ & $1.063 \mathrm{e}+0$ & $1.427 \mathrm{e}+0$ & 2.404 \\
32 & $4.492 \mathrm{e}-1$ & $2.533 \mathrm{e}-1$ & $5.424 \mathrm{e}-1$ & $6.993 \mathrm{e}-1$ & 2.358 \\
64 & $2.244 \mathrm{e}-1$ & $1.231 \mathrm{e}-1$ & $2.732 \mathrm{e}-1$ & $3.462 \mathrm{e}-1$ & 2.345 \\
128 & $1.121 \mathrm{e}-1$ & $6.112 \mathrm{e}-2$ & $1.370 \mathrm{e}-1$ & $1.722 \mathrm{e}-1$ & 2.341 \\
256 & $5.605 \mathrm{e}-2$ & $3.050 \mathrm{e}-2$ & $6.860 \mathrm{e}-2$ & $8.590 \mathrm{e}-2$ & 2.340 \\
\hline
\end{tabular}

TABLE 2. Comparison of the error estimators $\eta_{0}$ and $\eta_{\text {sym }}$ for Example 1.

\begin{tabular}{rccccccc}
\hline & \multicolumn{3}{c}{$\eta_{0}$} & & \multicolumn{3}{c}{$\eta_{s y m}$} \\
\cline { 2 - 4 } \cline { 6 - 8 } $1 / h$ & $\eta_{C F}$ & $\eta_{A S}$ & $\theta$ & & $\eta_{C F}$ & $\eta_{A S}$ & $\theta$ \\
\hline 8 & $1.573 \mathrm{e}+0$ & $3.918 \mathrm{e}+0$ & 3.468 & & $2.809 \mathrm{e}+0$ & $0.000 \mathrm{e}+0$ & 2.291 \\
16 & $5.631 \mathrm{e}-1$ & $2.085 \mathrm{e}+0$ & 3.333 & & $1.308 \mathrm{e}+0$ & $0.000 \mathrm{e}+0$ & 2.151 \\
32 & $2.533 \mathrm{e}-1$ & $1.064 \mathrm{e}+0$ & 3.310 & & $6.498 \mathrm{e}-1$ & $0.000 \mathrm{e}+0$ & 2.125 \\
64 & $1.231 \mathrm{e}-1$ & $5.359 \mathrm{e}-1$ & 3.307 & & $3.259 \mathrm{e}-1$ & $0.000 \mathrm{e}+0$ & 2.119 \\
128 & $6.112 \mathrm{e}-2$ & $2.688 \mathrm{e}-1$ & 3.308 & & $1.635 \mathrm{e}-1$ & $0.000 \mathrm{e}+0$ & 2.118 \\
256 & $3.050 \mathrm{e}-2$ & $1.346 \mathrm{e}-1$ & 3.309 & & $8.188 \mathrm{e}-2$ & $0.000 \mathrm{e}+0$ & 2.117 \\
\hline
\end{tabular}

Example 2. Let $(r, \theta)$ be the polar coordinates and consider the solution $\boldsymbol{u}=\left(u_{1}, u_{2}\right)$ on the $\Gamma$-shaped domain $\Omega=(-1,1)^{2} \backslash[0,1] \times[-1,0] \quad($ cf. $[2,3])$

$$
\begin{aligned}
& u_{1}(r, \theta)=\frac{1}{2 \mu} r^{\alpha}(A \cos (\alpha \theta)-\cos ((\alpha-2) \theta)), \\
& u_{2}(r, \theta)=\frac{1}{2 \mu} r^{\alpha}(B \sin (\alpha \theta)+\sin ((\alpha-2) \theta))
\end{aligned}
$$

with the parameters chosen as

$$
\mu=1.0, \quad \lambda=5.0, \quad \alpha=0.6, \quad A=1.0, \quad B=\frac{2(3 \mu+\lambda)}{(\lambda+\mu) \alpha}-A .
$$

The corresponding body force $f$ is then zero and the Dirichlet boundary condition is imposed on the whole boundary $\partial \Omega$.

As the solution $\boldsymbol{u}$ is singular near the origin, we perform adaptive mesh refinement starting with the initial mesh shown in Fig. 1 and using the maximum criterion: mark an element $T \in \mathcal{T}_{h}$ for refinement if

$$
\eta_{T}>0.5 \max _{T^{\prime} \in \mathcal{T}_{h}} \eta_{T^{\prime}}
$$

where the local error indicator $\eta_{T}$ is defined with the three contributions of $\eta_{1}$

$$
\eta_{T}:=\left(\eta_{C F, T}^{2}+\frac{R_{S}}{2 \mu}\left\|\boldsymbol{\sigma}_{h}^{A S}\right\|_{0, T}^{2}+\eta_{N C, T}^{2}\right)^{1 / 2} .
$$



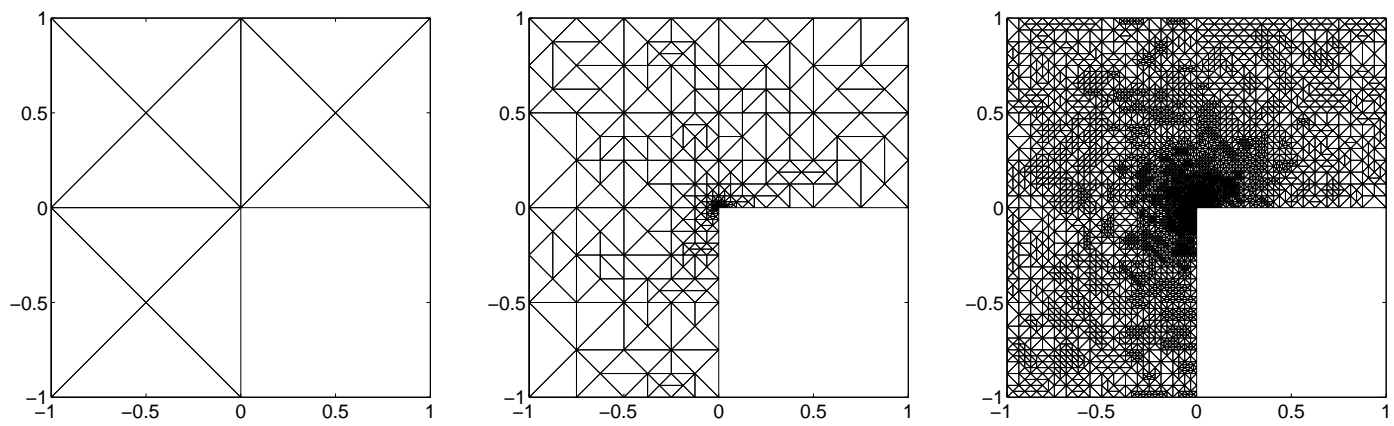

FIGURE 1. Initial and adapted meshes after 7 and 14 refinements for Example 2.

TABLE 3. Number of unknowns, actual error, three contributions of $\eta_{1}$ and effectivity index for Example 2.

\begin{tabular}{rccccc}
\hline$N$ & $\left\|\boldsymbol{u}-\boldsymbol{u}_{h}\right\|$ & $\eta_{C F}$ & $\eta_{A S}$ & $\eta_{N C}$ & $\theta$ \\
\hline 44 & $8.782 \mathrm{e}-1$ & $1.201 \mathrm{e}-1$ & $3.294 \mathrm{e}-1$ & $1.815 \mathrm{e}+0$ & 2.130 \\
132 & $6.379 \mathrm{e}-1$ & $1.535 \mathrm{e}-1$ & $3.371 \mathrm{e}-1$ & $1.552 \mathrm{e}+0$ & 2.551 \\
240 & $4.917 \mathrm{e}-1$ & $1.318 \mathrm{e}-1$ & $2.681 \mathrm{e}-1$ & $1.123 \mathrm{e}+0$ & 2.424 \\
364 & $3.840 \mathrm{e}-1$ & $1.190 \mathrm{e}-1$ & $2.192 \mathrm{e}-1$ & $8.363 \mathrm{e}-1$ & 2.349 \\
490 & $3.218 \mathrm{e}-1$ & $1.113 \mathrm{e}-1$ & $1.920 \mathrm{e}-1$ & $6.791 \mathrm{e}-1$ & 2.311 \\
686 & $2.714 \mathrm{e}-1$ & $1.005 \mathrm{e}-1$ & $1.724 \mathrm{e}-1$ & $5.377 \mathrm{e}-1$ & 2.222 \\
960 & $2.303 \mathrm{e}-1$ & $9.114 \mathrm{e}-2$ & $1.542 \mathrm{e}-1$ & $4.567 \mathrm{e}-1$ & 2.251 \\
1432 & $1.845 \mathrm{e}-1$ & $7.667 \mathrm{e}-2$ & $1.307 \mathrm{e}-1$ & $3.721 \mathrm{e}-1$ & 2.309 \\
2102 & $1.498 \mathrm{e}-1$ & $6.570 \mathrm{e}-2$ & $1.137 \mathrm{e}-1$ & $3.101 \mathrm{e}-1$ & 2.391 \\
3472 & $1.185 \mathrm{e}-1$ & $5.214 \mathrm{e}-2$ & $9.058 \mathrm{e}-2$ & $2.411 \mathrm{e}-1$ & 2.364 \\
4998 & $9.802 \mathrm{e}-2$ & $4.403 \mathrm{e}-2$ & $7.717 \mathrm{e}-2$ & $2.014 \mathrm{e}-1$ & 2.398 \\
7400 & $8.015 \mathrm{e}-2$ & $3.718 \mathrm{e}-2$ & $6.491 \mathrm{e}-2$ & $1.671 \mathrm{e}-1$ & 2.444 \\
11618 & $6.473 \mathrm{e}-2$ & $2.970 \mathrm{e}-2$ & $5.166 \mathrm{e}-2$ & $1.322 \mathrm{e}-1$ & 2.398 \\
17010 & $5.324 \mathrm{e}-2$ & $2.468 \mathrm{e}-2$ & $4.281 \mathrm{e}-2$ & $1.094 \mathrm{e}-1$ & 2.414 \\
25886 & $4.288 \mathrm{e}-2$ & $2.029 \mathrm{e}-2$ & $3.545 \mathrm{e}-2$ & $8.919 \mathrm{e}-2$ & 2.453 \\
39068 & $3.503 \mathrm{e}-2$ & $1.665 \mathrm{e}-2$ & $2.896 \mathrm{e}-2$ & $7.285 \mathrm{e}-2$ & 2.454 \\
\hline
\end{tabular}

Two adapted meshes generated after 7 and 14 refinements are shown in the middle and right figures of Fig. 1. As expected, the mesh refinement is highly concentrated around the origin to resolve the singular behavior of $\boldsymbol{u}$. Furthermore, numerical results reported in Tables 3-4 confirm the theory and the effectiveness of our estimator $\eta_{1}$ also in the context of adaptive mesh refinement. Finally, it is found that the actual error attains the optimal order of convergence with respect to the number of unknowns $N$

$$
\left\|\boldsymbol{u}-\boldsymbol{u}_{h}\right\|=O\left(N^{-0.50}\right),
$$

demonstrating the efficiency of adaptive mesh refinement. 
TABLE 4. Comparison of the error estimators $\eta_{0}$ and $\eta_{s y m}$ for Example 2.

\begin{tabular}{rccccccc}
\hline & \multicolumn{4}{c}{$\eta_{0}$} & & \multicolumn{3}{c}{$\eta_{\text {sym }}$} \\
\cline { 2 - 4 } \cline { 7 - 8 }$N$ & $\eta_{C F}$ & $\eta_{A S}$ & $\theta$ & & $\eta_{C F}$ & $\eta_{A S}$ & $\theta$ \\
\hline 44 & $1.201 \mathrm{e}-1$ & $6.461 \mathrm{e}-1$ & 2.242 & & $6.056 \mathrm{e}-1$ & $0.000 \mathrm{e}+0$ & 2.179 \\
132 & $1.535 \mathrm{e}-1$ & $6.612 \mathrm{e}-1$ & 2.743 & & $5.243 \mathrm{e}-1$ & $0.000 \mathrm{e}+0$ & 2.568 \\
240 & $1.318 \mathrm{e}-1$ & $5.259 \mathrm{e}-1$ & 2.639 & & $4.091 \mathrm{e}-1$ & $0.000 \mathrm{e}+0$ & 2.430 \\
364 & $1.190 \mathrm{e}-1$ & $4.299 \mathrm{e}-1$ & 2.595 & & $3.242 \mathrm{e}-1$ & $0.000 \mathrm{e}+0$ & 2.336 \\
490 & $1.113 \mathrm{e}-1$ & $3.767 \mathrm{e}-1$ & 2.584 & & $2.818 \mathrm{e}-1$ & $0.000 \mathrm{e}+0$ & 2.285 \\
686 & $1.005 \mathrm{e}-1$ & $3.382 \mathrm{e}-1$ & 2.541 & & $2.503 \mathrm{e}-1$ & $0.000 \mathrm{e}+0$ & 2.185 \\
960 & $9.114 \mathrm{e}-2$ & $3.025 \mathrm{e}-1$ & 2.603 & & $2.203 \mathrm{e}-1$ & $0.000 \mathrm{e}+0$ & 2.202 \\
1432 & $7.667 \mathrm{e}-2$ & $2.564 \mathrm{e}-1$ & 2.690 & & $1.827 \mathrm{e}-1$ & $0.000 \mathrm{e}+0$ & 2.247 \\
2102 & $6.570 \mathrm{e}-2$ & $2.229 \mathrm{e}-1$ & 2.814 & & $1.527 \mathrm{e}-1$ & $0.000 \mathrm{e}+0$ & 2.307 \\
3472 & $5.214 \mathrm{e}-2$ & $1.777 \mathrm{e}-1$ & 2.797 & & $1.215 \mathrm{e}-1$ & $0.000 \mathrm{e}+0$ & 2.278 \\
4998 & $4.403 \mathrm{e}-2$ & $1.514 \mathrm{e}-1$ & 2.851 & & $1.021 \mathrm{e}-1$ & $0.000 \mathrm{e}+0$ & 2.304 \\
7400 & $3.718 \mathrm{e}-2$ & $1.273 \mathrm{e}-1$ & 2.915 & & $8.438 \mathrm{e}-2$ & $0.000 \mathrm{e}+0$ & 2.336 \\
11618 & $2.970 \mathrm{e}-2$ & $1.013 \mathrm{e}-1$ & 2.864 & & $6.769 \mathrm{e}-2$ & $0.000 \mathrm{e}+0$ & 2.294 \\
17010 & $2.468 \mathrm{e}-2$ & $8.397 \mathrm{e}-2$ & 2.885 & & $5.581 \mathrm{e}-2$ & $0.000 \mathrm{e}+0$ & 2.306 \\
25886 & $2.029 \mathrm{e}-2$ & $6.953 \mathrm{e}-2$ & 2.941 & & $4.560 \mathrm{e}-2$ & $0.000 \mathrm{e}+0$ & 2.336 \\
39068 & $1.665 \mathrm{e}-2$ & $5.680 \mathrm{e}-2$ & 2.943 & & $3.715 \mathrm{e}-2$ & $0.000 \mathrm{e}+0$ & 2.334 \\
\hline
\end{tabular}

\section{REFERENCES}

[1] R. Verfürth, A review of a posteriori error estimation techniques for elasticity problems, Comput. Methods Appl. Mech. Engrg., 176 (1999), 419-440.

[2] S. Nicaise, K. Witowski and B.I. Wohlmuth, An a posteriori error estimator for the Lamé equation based on equilibrated fluxes, IMA J. Numer. Anal., 28 (2008), 331-353.

[3] M. Ainsworth and R. Rankin, Guaranteed computable error bounds for conforming and nonconforming finite element analyses in planar elasticity, Internat. J. Numer. Methods Engrg., 82 (2010), 1114-1157.

[4] H.-C. Lee and K.-Y. Kim, A posteriori error estimators for stabilized P1 nonconforming approximation of the Stokes problem, Comput. Methods Appl. Mech. Engrg., 199 (2010), 2903-2912.

[5] P. Hansbo and M.G. Larson, Discontinuous Galerkin and the Crouzeix-Raviart element: application to elasticity, M2AN Math. Model. Numer. Anal., 37 (2003), 63-72.

[6] C.O. Horgan and L.E. Payne, On inequalities of Korn, Friedrichs and Babuška-Aziz, Arch. Rational Mech. Anal., 82 (1983), 165-179.

[7] C.O. Horgan, Korn's inequalities and their applications in continuum mechanics, SIAM Rev., 37 (1995), 491-511.

[8] K.-Y. Kim, Guaranteed a posteriori error estimator for mixed finite element methods of linear elasticity with weak stress symmetry, submitted for publication.

[9] M. Bebendorf, A note on the Poincaré inequality for convex domains, Z. Anal. Anwendungen, 22 (2003), 751-756.

[10] M. Ainsworth and J.T. Oden, A posteriori error estimation in finite element analysis, John Wiley and Sons, New York, 2000.

[11] S. Ohnimus, E. Stein and E. Walhorn, Local error estimates of FEM for displacements and stresses in linear elasticity by solving local Neumann problems, Internat. J. Numer. Methods Engrg., 52 (2001), 727-746.

[12] K.-Y. Kim, Flux reconstruction for the P2 nonconforming finite element method with application to a posteriori error estimation, submitted for publication. 